\section{In Leishmaniasis Due to Leishmania guyanensis Infection, Distinct Intralesional Interleukin-10 and Foxp3 mRNA Expression Are Associated with Unresponsiveness to Treatment}

\author{
Eliane Bourreau, ${ }^{1, a}$ Catherine Ronet, ${ }^{3, a}$ Edith Darsissac, ${ }^{1}$ \\ Marie-Claire Lise, ${ }^{1}$ Dominique Sainte Marie, ${ }^{2}$ Emmanuel Clity, ${ }^{2}$ \\ Fabienne Tacchini-Cottier, ${ }^{3}$ Pierre Couppie, ${ }^{2}$ and Pascal Launois ${ }^{1,3}$ \\ ${ }^{1}$ Immunologie des Leishmanioses, Institut Pasteur de la Guyane, and 2Institut \\ Guyanais de Dermatologie Tropicale, EA 2188, Centre Hospitalier Andrée \\ Rosemon, Cayenne, French Guyana; ${ }^{3}$ World Health Organization Immunology \\ Research and Training Centre, Department of Biochemistry, University of \\ Lausanne, Epalinges, Switzerland
}

The presence of intralesional natural regulatory $\mathrm{T}$ cells, characterized by the expression of Foxp 3 mRNA, was analyzed in patients with localized leishmaniasis due to Leishmania guyanensis infection that was unresponsive to treatment with pentamidine isethionate. Foxp3 mRNA levels were associated with unresponsiveness to treatment among patients with a lesion duration of $\geqslant 1$ month, but this association was not observed among patients with a lesion duration of $<1$ month. In conclusion, high intralesional expression of Foxp3 might be an indicator of poor response to treatment, depending on the duration of lesions.

Infections with Leishmania species induce a spectrum of diseases, from self-healing cutaneous leishmaniasis to mucosal and visceral leishmaniasis. The clinical outcome depends on the infective agent and on the specific immune response to Leishmania antigens.

Given the great variety of epidemiological situations, the different factors influencing disease transmission, and the incomplete knowledge of the biology of the parasite and its vector and

Received 8 July 2008; accepted 15 September 2008; electronically published 6 January 2009.

Potential conflicts of interest: none reported.

Financial support: Institute Pasteur; French Ministry of Research; Swiss National Foundation (grant 310000-107719).

a E.B. and C.R. contributed equally to this work.

Reprints or correspondence: Prof. P. Launois, WHO-IRTC, Dept. of Biochemistry, University of Lausanne, Ch des boveresses 155, 1066 Epalinges, Switzerland (p.launois@unil.ch).

The Journal of Infectious Diseases 2009; 199:576-9

(C) 2009 by the Infectious Diseases Society of America. All rights reserved. 0022-1899/2009/19904-0016\$15.00

DOI: $10.1086 / 596508$ reservoirs, disease control is difficult to achieve. Furthermore, effective prevention is difficult to achieve, and current curative therapy is costly, often poorly tolerated, and not always effective.

Failure of antileishmanial therapy (such as antimonials, pentamidine, and amphotericin) is often due to drug resistance. There is currently no markers of resistance to leishmanicidal drugs, and the only method for monitoring resistance relies on an in vitro amastigote/macrophage system [1].

It is fully accepted that the leishmanicidal effect of conventional therapy (pentavalent antimony and pentamidine) for experimental visceral leishmaniasis requires $\mathrm{T}$ cells, interferon (IFN) $-\gamma$, and interleukin (IL)-12 [2-5]. In addition, in a randomized, controlled trial, the cure rate with antimonials treatment was reported to be lower in HIV-infected patients with visceral leishmaniasis than in non-HIV-infected patients [6], suggesting that cellular immune responses play an important role in unresponsiveness to treatment during human infection.

In this context, we have already demonstrated that high intralesional IL-10 mRNA expression is associated with unresponsiveness to treatment with pentamidine isethionate during the early phases of infection with $L$. guyanensis (i.e., $<30$ days of evolution) [7]. IL-10 might regulate the responses to chemotherapy by inhibiting IL-12 and/or IFN- $\gamma$ production.

Among the populations of cells that can produce IL-10, the recently described regulatory $T\left(T_{\text {reg }}\right)$ cells have been extensively analyzed in the context of tolerance, autoimmunity, and infections $[8,9]$. These cells specifically express the transcription factor Foxp3 [10]. During cutaneous infection with Leishmania braziliensis, human $\mathrm{T}_{\text {reg }}$ cells have been shown to accumulate at the site of infection and contribute to the control of effector $\mathrm{T}$ cell functions [11]. However, the mechanisms of such suppression are debatable. Indeed, although the cytokines they produce (i.e., IL-10 and transforming growth factor [TGF] $-\beta$ ) have been described as suppressive, recent data have clearly shown that, in both human and experimental infection, the IL-10 produced by $\mathrm{T}_{\text {reg }}$ cells is not necessary for the suppressive activity of these cells [12-14].

In the present study, we investigated whether, in patients infected with L. guyanensis, the presence of $\mathrm{T}_{\text {reg }}$ cells, characterized by the expression of Foxp3 mRNA, is associated with unresponsiveness to treatment.

Methods. Forty-eight patients (36 males and 12 females who were 16-57 years old) with localized cutaneous leishmaniasis due to L. guyanensis infection underwent precise clinical examination of the lesions, including localization and enumeration, before treatment with pentamidine isethionate (Penta- 
carinat; Rhone Poulenc), which was administrated at $4 \mathrm{mg} / \mathrm{kg}$ in 2 intramuscular injections on days 1 and 3. All patients were seronegative for HIV.

Samples from patients with localized cutaneous leishmaniasis were collected on days 15-150 of lesion evolution. Eleven and 37 patients developed lesions for $<1$ and $\geqslant 1$ month, respectively. Informed consent was obtained from the patients, and the human experimentation guidelines of the Centre Hospitalier Andrée Rosemon in Cayenne were followed in the conduct of this research.

Parasitological diagnosis was done by collecting serous dermal fluid from the border of the lesion, using May-GrunwaldGiemsa staining. Parasites isolates were determined to be L. guyanensis on the basis of isoenzyme polymorphism on parasite culture.

Clinical evaluation after treatment (between 1 and 3 months) showed that lesions of 37 of the 48 patients had regressed (group 1 patients, responsive to treatment), whereas lesions of 11 patients were unchanged or worsened (group 2 patients, unresponsive to treatment). Of the 11 patients unresponsive to treatment, 3 developed lesions for $<1$ month, and 8 developed lesions for $\geqslant 1$ month. These patients were given a second treatment with pentamidine isothionate at the same dose.

Punch biopsies (2-mm diameter) were done and total RNA was isolated as described elsewhere [7], using the RNeasy Mini Kit (Qiagen). First-strand cDNA synthesis was done on total RNA by means of a first-strand cDNA synthesis kit (Superscript III First-Strand Synthesis System; Invitrogen). Real-time polymerase chain reaction was performed using the 7300 Real-Time System (Applied Biosystems). FAM-MGB-labeled primer/ probe sets for IFN- $\gamma$ (Hs 00174143-m1), IL-10 (Hs00174086m1), Foxp3 (Hs 00203958-m1), and $\beta$-actin (Hs 99999903-m1; used as endogenous control) were from Applied Biosystems. The relative quantification of products was determined by the comparative threshold cycle $\left(\mathrm{C}_{\mathrm{t}}\right)$ method, using the equation

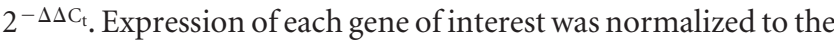
expression of the $\beta$-actin endogenous gene, and the results are displayed as relative to the expression of the same gene in peripheral blood mononuclear cells. In some experiments, results are expressed as the fold change of the specific gene in lesions of patients unresponsive to treatment compared with patients responsive to treatment, which was defined as the reference. For statistical analysis, we used the Wilcoxon rank sum test to compare levels of cytokine mRNA within the different groups of patients.

Results. We previously demonstrated that, during the first month of lesion duration, high intralesional IL-10 mRNA expression is associated with unresponsiveness to treatment with pentamidine isothionate [7]. To determine whether this IL-10 mRNA expression is associated with Foxp3 mRNA expression, we analyzed samples from 11 patients with a lesion duration of $<1$ month. Three of these patients who were unresponsive to treatment showed a high level of IL-10 mRNA expression (group $2, n=3$ ) (figure $1 A$ ), confirming our previous result. However, among patients with a lesion duration of $<1$ month, equivalent Foxp3 mRNA expression was detected in patients responsive (group 1, $n=8$ ) or unresponsive (group 2, $n=3$ ) to treatment (figure $1 A$ ). In addition, IFN- $\gamma$ levels were not significantly different between both groups of patients (figure $1 A$ ).

Among patients with a lesion duration of $\geqslant 1$ month, levels of Foxp3 mRNA transcripts were significantly higher in skin lesions of patients unresponsive to treatment (group 2, $n=8$ ) than in those of patients responsive to treatment (group 1, $n=29)(P<.04)$ (figure $1 B)$. The mean \pm SD fold increase in Foxp3 mRNA expression in patients from group 2 compared with those from group 1 was $2.1 \pm 0.32$ (figure $1 C$ ). In contrast, levels of IL-10 mRNA were identical in patients responsive or unresponsive to treatment (figure $1 B$ and $1 C$ ), and levels of IFN- $\gamma$ mRNA transcripts were significantly lower in patients unresponsive to treatment than in patients responsive to treatment $(P<.007)$ (figure $1 B)$. Indeed, IFN- $\gamma$ mRNA expression in $\mathrm{pa}$ tients from group 2 was 4 -fold less than that in patients from group 1 (figure $1 C$ ). IL-12p40 mRNA was not detectable in lesions of patients responsive or unresponsive to treatment (data not shown).

Discussion. In this work, we have demonstrated that, in patients with a lesion duration of $\geqslant 1$ month, unresponsiveness to treatment was associated with high levels of Foxp3 mRNA at the site of infection. Given that Foxp3 is a specific transcription factor of $\mathrm{T}_{\text {reg }}$ cells [10], these results strongly suggest that unresponsiveness to treatment may be associated with a local suppression of immune responses mediated by $\mathrm{T}_{\text {reg }}$ cells.

Because a functional immune response is required for successful treatment, we postulate that, in patients with a lesion duration of $\geqslant 1$ month, $\mathrm{T}_{\text {reg }}$ cells may regulate IL-12 or IFN- $\gamma$ production and/or activity. Interestingly, although we were unable to detect IL-12p40 in the skin lesions of patients with a lesion duration of $\geqslant 1$ month whether responsive or unresponsive to treatment, we clearly demonstrated that IFN- $\gamma$ transcripts in lesions were lower among patients unresponsive to treatment than among patients responsive to treatment. These results suggest that intralesional $\mathrm{T}_{\text {reg }}$ cells have suppressive functions, as previously described in patients infected with $L$. braziliensis [11].

Because an IL-10 - dependent suppressive role of $\mathrm{T}_{\text {reg }}$ cells has been reported, we analyzed IL-10 mRNA expression in patients with a lesion duration of $\geqslant 1$ month who were unresponsive to treatment. No significant increase the level of IL-10 mRNA transcripts was demonstrated in these patients, compared with that in patients responsive to treatment, suggesting that suppression induced by $\mathrm{T}_{\text {reg }}$ cells is independent of IL-10. This is in accordance with the findings of previous reports showing that, in either human or experimental cutaneous and visceral leishmaniasis, the IL-10 produced by Foxp $3^{+}$T cells is not associated with 

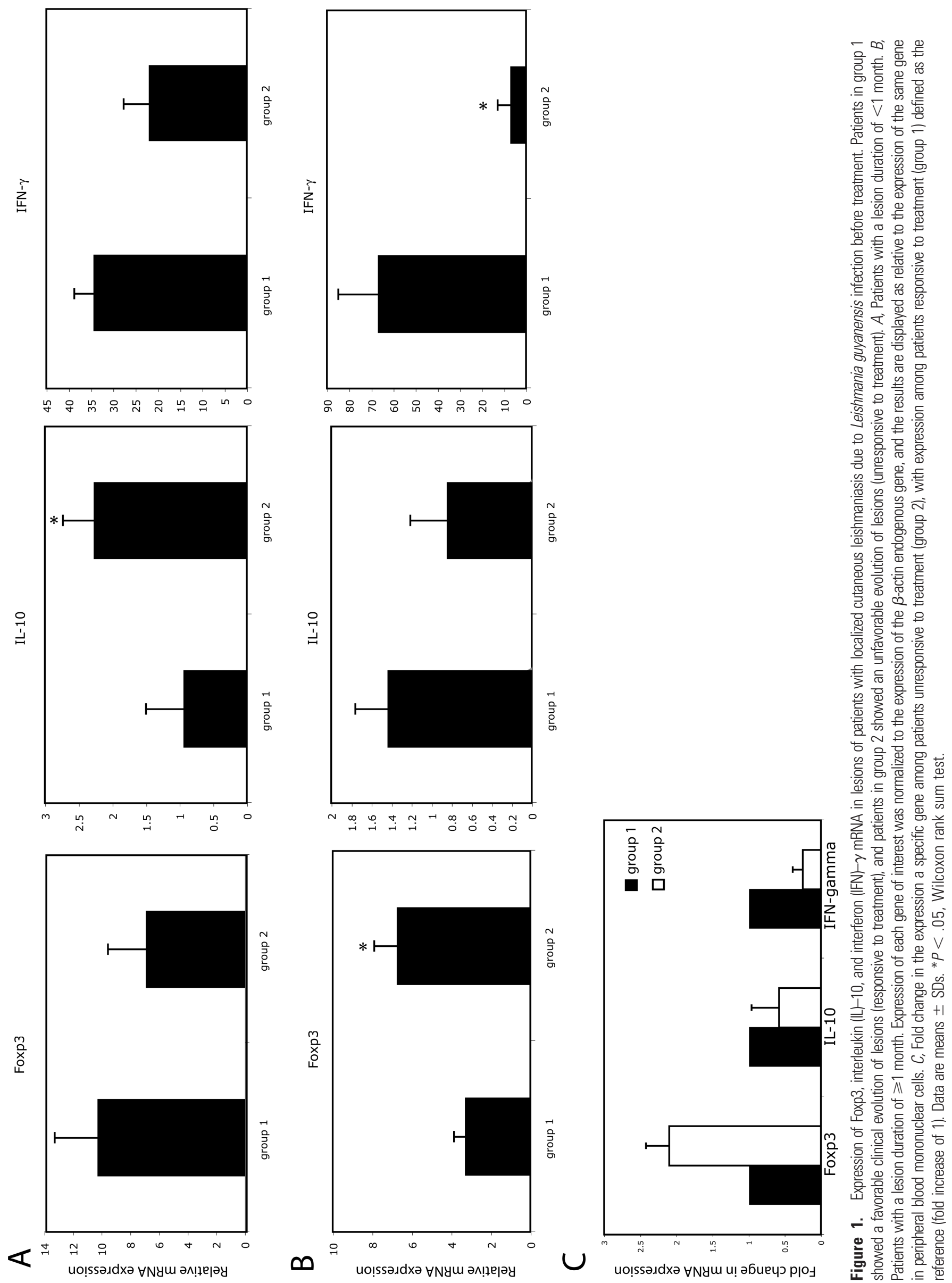
the immunosuppression described during Leishmania infection [12-14].

TGF- $\beta$, another cytokine produced by $\mathrm{T}_{\text {reg }}$ cells, is also involved in the immunosuppression mediated by $\mathrm{T}_{\text {reg }}$ cells. However, we were unable to detect TGF- $\beta$ production by $\mathrm{CD} 4{ }^{+} \mathrm{CD} 25^{+} \mathrm{T}$ cells isolated from biopsy samples and stimulated in vitro by L. guyanensis (data not shown). Nonetheless, we cannot exclude a role for this cytokine-particularly its membrane form-in the immunosuppression induced by $\mathrm{T}_{\text {reg }}$ cells.

Immune responses induced by L. guyanensis have been shown to be distinct during the early phase ( $<1$ month of lesion duration) versus the late phase ( $\geqslant 1$ month of lesion duration). Indeed, Th2 and Th1 responses predominate during the early and late phases of infection, respectively [15]. In line with this, we confirmed in the present work our previous results showing that, among patients with a lesion duration of $<1$ month, high intralesional IL-10 mRNA expression is associated with unresponsiveness to treatment with pentamidine isothionate [7]. Thus, to determine if IL-10 - producing cells are $\mathrm{T}_{\text {reg }}$ cells, Foxp3 mRNA expression was analyzed in patients with a lesion duration of $<1$ month. Although IL-10 mRNA expression was higher among patients unresponsive to treatment than among to patients responsive to treatment, Foxp3 mRNA expression did not differ between patients responsive or unresponsive to treatment. Interestingly, altogether these results confirm the dichotomy of the 2 phases (early versus late) of infection with L. guyanensis.

In conclusion, unresponsiveness to treatment is associated with high IL-10 and not Foxp3 mRNA expression at the site of infection among patients with a lesion duration of $<1$ month. In contrast, among patients with a lesion duration of $\geqslant 1$ month, high Foxp3 but not IL-10 mRNA expression is associated with unresponsiveness to treatment, although the exact mechanisms involved in the suppression of $\mathrm{T}_{\text {reg }}$ cells has not yet been elucidated. However, these markers (i.e., high level of Foxp3 or IL-10 mRNA expression) should be tools of predictive value for poor responsiveness to treatment (in addition to time of lesion duration) and could influence the therapeutic scheme of treatment.

\section{References}

1. Croft SL, Sundar S, Fairlamb AH. Drug resistance in leishmaniasis. Clin Microbiol Rev 2006; 19:111-26.

2. Murray HW, Delph-Etienne S. Roles of endogenous gamma interferon and macrophage microbicidal mechanisms in host response to chemotherapy in experimental visceral leishmaniasis. Infect Immun 2000; 68: 288-93.

3. Murray HW, Hariprashad J, Fichtl RE. Treatment of experimental visceral leishmaniasis in a T-cell-deficient host: response to amphotericin B and pentamidine. Antimicrob Agents Chemother 1993; 37:1504-5.

4. Murray HW, Oca MJ, Granger AM, Schreiber RD. Requirement for T cells and effect of lymphokines in successful chemotherapy for an intracellular infection: experimental visceral leishmaniasis. J Clin Invest 1989; 83:1253-7.

5. Murray HW, Berman JD, Wright SD. Immunochemotherapy for intracellular Leishmania donovani infection: gamma interferon plus pentavalent antimony. J Infect Dis 1988; 157:973-8.

6. Laguna F, Lopez-Velez R, Pulido F, et al. Treatment of visceral leishmaniasis in HIV-infected patients: a randomized trial comparing meglumine antimoniate with amphotericin B. Spanish HIV-Leishmania Study Group. AIDS 1999; 13:1063-9.

7. Bourreau E, Prevot G, Gardon J, Pradinaud R, Launois P. High intralesional interleukin-10 messenger RNA expression in localized cutaneous leishmaniasis is associated with unresponsiveness to treatment. J Infect Dis 2001 ; $184: 1628-30$.

8. Shevach EM. CD4 ${ }^{+} \mathrm{CD} 25^{+}$suppressor T cells: more questions than answers. Nat Rev Immunol 2002; 2:389-400.

9. Rouse BT, Suvas S. Regulatory cells and infectious agents: detentes cordiale and contraire. J Immunol 2004; 173:2211-5.

10. Hori S, Nomura T, Sakaguchi S. Control of regulatory T cell development by the transcription factor Foxp3. Science 2003; 299:1057-61.

11. Campanelli AP, Roselino AM, Cavassani KA, et al. CD $4^{+} \mathrm{CD} 25^{+} \mathrm{T}$ cells in skin lesions of patients with cutaneous leishmaniasis exhibit phenotypic and functional characteristics of natural regulatory T cells. J Infect Dis 2006; 193:1313-22.

12. Anderson CF, Oukka M, Kuchroo VJ, Sacks D. CD $4^{+} \mathrm{CD} 25^{-}$Foxp $3^{-}$ Th1 cells are the source of IL-10-mediated immune suppression in chronic cutaneous leishmaniasis. J Exp Med 2007; 204:285-97.

13. Nagase H, Jones KM, Anderson CF, Noben-Trauth N. Despite increased $\mathrm{CD}^{+}{ }^{+}$Foxp $^{+}$cells within the infection site, BALB/c IL-4 receptordeficient mice reveal CD $4^{+}$Foxp $3^{-}$negative T cells as a source of IL-10 in Leishmania major susceptibility. J Immunol 2007; 179:2435-44.

14. Nylen S, Maurya R, Eidsmo L, Manandhar KD, Sundar S, Sacks D. Splenic accumulation of IL-10 mRNA in $\mathrm{T}$ cells distinct from $\mathrm{CD} 4{ }^{+} \mathrm{CD} 25^{+}$(Foxp3) regulatory $\mathrm{T}$ cells in human visceral leishmaniasis. J Exp Med 2007; 204:805-17.

15. Bourreau E, Gardon J, Pradinaud R, et al. Th2 responses predominate during the early phases of infection in patients with localized cutaneous leishmaniasis and precede the development of Th1 responses. Infect Immun 2003; 71:2244-6. 